\title{
Nuevos instrumentos de política macroprudencial de la Unión Europea
}

\author{
Valeria Ivett Luna Flores*
}

En el verano del año 2010 el Consejo General del Banco Central Europeo decidió que el conjunto de 27 bancos centrales de la Unión Europea participaran conjuntamente en una investigación formulando nuevos conceptos, modelos y medidas de regulación y supervisión macroprudencial. El grupo de investigación macroprudencial $^{1}$ trabajó conjuntamente durante 4 años, realizando 161 documentos de investigación ${ }^{2}$ y los resultados finales de este trabajo se dieron a conocer en el sitio del Banco Central Europeo en junio de 2014 en un documento titulado Final Results of Macro-prudential Research Network.

Este trabajo se divide en tres secciones. En la primera sección se presentan cuáles son los temas que investigaron cada uno de los tres grupos de trabajo así como las preguntas por responder. En la sección II se presentan dos de los nuevos indicadores macroprudenciales [uno relacionado con el estrés sistémico llamado indicador compuesto de tensiones sistémicas (CIss) y otro relacionado con la integración financiera conocido con el nombre de indicador de integración

1 En inglés: Macro-prudential Research Network (MaRs)

${ }^{2}$ Lista de los trabajos de investigación del grupo Macro-prudential Research Network publicados: https://www.ecb.europa.eu/home/html/ researcher_mars.en.html financiera (SYNFINT)]. Al final se dan a

conocer las principales conclusiones.

\section{Sección I.}

\section{Temáticas y preguntas}

Cada uno de los grupos de trabajo analizó una temática diferente

1. Modelos macrofinancieros y estabilidad. Este grupo realizó una profunda investigación para contar con herramientas analíticas importantes que pudieran ser usadas regularmente $\mathrm{y}$ se enfocó en el desarrollo de modelos macroeconómicos diseñados para evaluar los instrumentos de política macroprudencial. La investigación de este grupo consistió en integrar una clase de inestabilidad financiera a gran escala en los modelos macroeconómicos utilizados por los bancos centrales. Este grupo elaboró un total de 65 trabajos de investigación. Se obtuvo un modelo macroeconómico que evalúa las políticas de regulación macroprudencial. De los 65 trabajos realizados sólo 30 han sido publicados. A este grupo se le conoce con el nombre de WS1. ${ }^{3}$

${ }^{3}$ Existe también otro grupo, conocido como WS2 que tuvo a su cargo la formulación de indicadores de riesgo sistémico. Fueron publicados 51 nuevos trabajos de investigación. Se realizó una evaluación comparativa de diferentes modelos que lograrán explicar las crisis financieras de los países de la Unión Europea.

\footnotetext{
*Estudiante de la Especialización "Economía Monetaria y Financiera”, unAm. Titular de un diploma de Doctorado en "Economía Monetaria y Bancaria” y de un Diplôme d’Etudes Approfondies, "Moneda y Finanzas" de la Universidad de Paris X, Nanterre, Francia. Email: luflovaleria@gmail.com
} 
2. Evaluación de riesgos de contagio. El grupo WS3 analizó los posibles mecanismos de riesgos de contagio. Dentro de este grupo se elaboraron 45 trabajos de investigación. Se analizaron las estructuras del mercado interbancario del euro para evaluar el efecto contagio de los riesgos usando la base de datos del sistema de pagos TARGET2. ${ }^{4}$

Sólo 72 de estos trabajos han sido publicados; el resto de las investigaciones está aún en la etapa de poder ser publicadas. Los resultados de este importante trabajo de investigación se dieron a conocer en tres conferencias públicas, una organizada en octubre de 2011, otra en octubre de 2012 y la última en junio de 2014. En cada conferencia se presentaron los mejores trabajos y colaboraciones de países extranjeros.

A lo largo de estos 161 trabajos de investigación se puede encontrar la respuesta a las siguientes preguntas que el Consejo General del BCE formuló: a) ¿cómo puede ser representada la inestabilidad en los modelos económicos a nivel agregado? b) ¿cómo afecta la estabilidad financiera a la economía real? c) ¿cuáles son los principales canales de transmisión de la inestabilidad financiera a nivel agregado? d) ¿cuál es el papel de los efectos no lineales de las variables y cuáles son los efectos de retroalimentación?

\footnotetext{
${ }^{4}$ Se trata de un sistema de pagos interbancario entre los países de la Unión Europea.
}

e) ¿cuáles son los efectos acumulados de la interacción entre la inestabilidad financiera y la eficiencia de la economía? f) ¿cómo puede ser descrito el ciclo de apalancamiento tanto a nivel teórico como empírico? g) ¿cómo explican estos modelos las causas y características de la reciente crisis? y $h$ ) ¿en qué medida estos nuevos modelos, identifican las políticas macroprudenciales apropiadas para mantener la estabilidad?

\section{Sección II. Principales resultados}

1. Nuevos indicadores de riesgo sistémico. Uno de los indicadores más importantes para medir el riesgo sistémico es el indicador compuesto de tensiones sistémicas [en inglés: Composite Indicator of Systemic Stress (CISs)] fue elaborado en el año 2012 por Kremer y Lo Duca (BCE) y Holló (Banco Central de Hungría). El BCE lo publica desde el año 2012 pero hay una serie histórica desde enero de 1999. El CISs incluye 15 indicadores de estrés ${ }^{5}$

\footnotetext{
${ }^{5}$ La volatilidad de la tasa de interés Euribor, el spread del mercado monetario, el volumen de préstamo marginal del $\mathrm{BCE}$, la volatilidad de los bonos gubernamentales, el estrés de los bonos no financieros, el spread de las tasas de los swaps, la volatilidad de la bolsa de valores del sector no financiero, la correlación de los bonos gubernamentales, la volatilidad de las acciones del sector bancario, el spread entre los bonos financieros y no financieros, la volatilidad de la paridad eurodólar, la volatilidad de la paridad euro-yen, la volatilidad de la paridad euro-libra esterlina, entre otros indicadores de estrés.
} 
de 5 mercados financieros: los intermediarios financieros, el mercado monetario, el mercado de acciones, el mercado de bonos y el mercado cambiario. El valor que toma este indicador va de 0 a 1 ; el valor de 1 indica un gran riesgo sistémico.

La aportación del ciss es que mide tanto el riesgo sistémico acumulado como la interrelación en términos de riesgo sistémico de cada uno de los mercados analizados. Ha llegado a ser uno de los indicadores más seguidos por las autoridades y agentes económicos. Hay varias versiones del cIss: a nivel de la Zona Euro se publica diariamente y mensualmente y sólo se publica, en frecuencia mensual, un indicador ciss para cada uno de los siguientes países: Alemania, Austria, Bélgica, Checoslovaquia, Dinamarca, España, Finlandia, Francia, Inglaterra, Grecia, Hungría, Irlanda, Italia, Holanda, Polonia, Portugal y Suecia.

Como se observa en la gráfica 1, el Ciss a principios del mes de diciembre de 2014 permanece al mismo nivel que tenía antes de la crisis financiera de 2008, un nivel menor a 0.3 a pesar de la actual volatilidad en los mercados cambiario, del petróleo y de materias primas. Este indicador tiene la capacidad de predecir una crisis como la del año 2008-2009, donde su valor alcanzó un valor superior a 0.8 .

\section{Gráfica I}

Indicador Compuesto de Tensiones Sistémicas, cIss

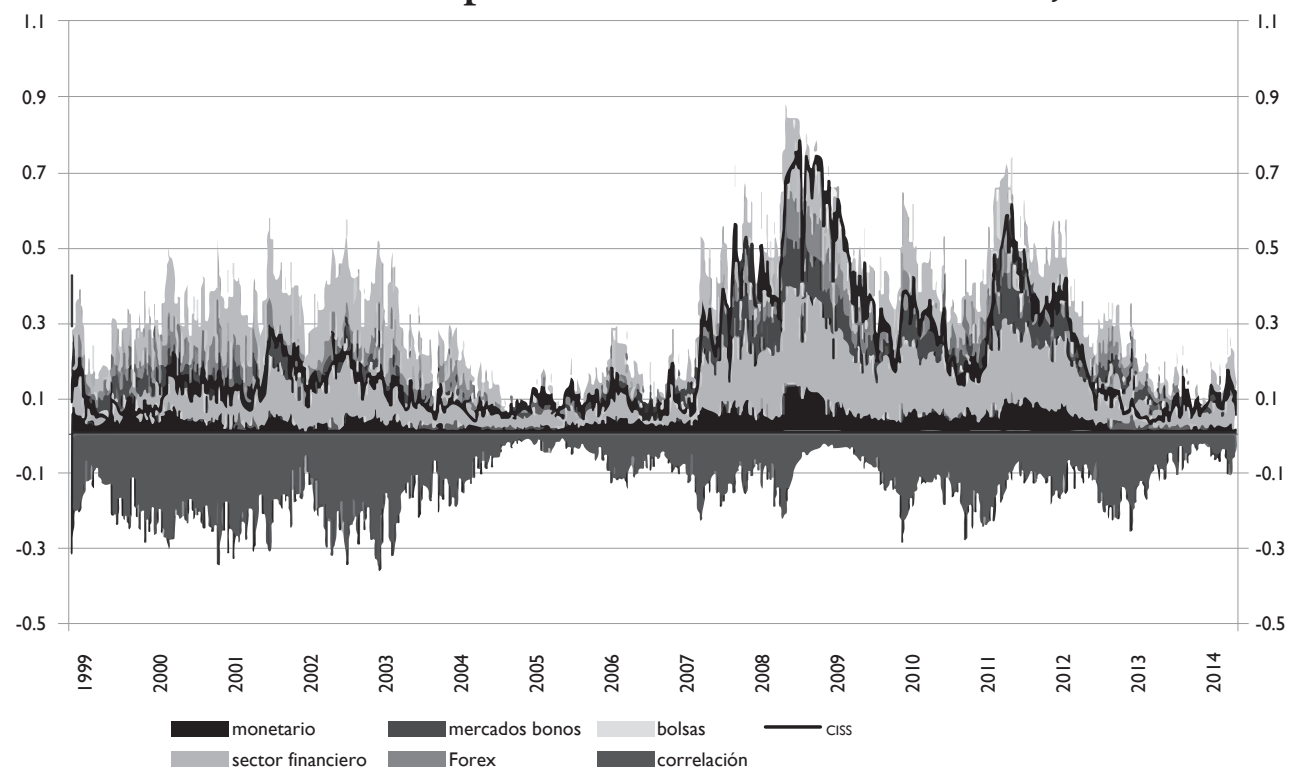

Fuente: ECB, período del 8 de enero de 1999 al 5 de diciembre de 2014. 
La construcción del indicador compuesto de tensiones sistémicas se basa en: a) la selección de un número de índices subyacentes que reflejen la variedad de las vulnerabilidades financieras, b) la estandarización de índices subyacentes que permitan relacionar a un conjunto de valores, y c) la agregación de los índices subyacentes estandarizados para obtener un indicador sintético único.

Por su parte, los índices subyacentes tienen como objetivo medir los fenómenos que revelan tensiones como la búsqueda de la calidad, la búsqueda de la liquidez o la incertidumbre de los agentes, describen el estrés con ayuda del análisis de la tendencia, de medidas de volatilidad y de estrategias de substitución de activos financieros que reflejen la actividad de los mercados financieros y el comportamiento de los agentes. Es preferible que estos índices subyacentes sean construidos con datos semanales o mensuales. Los subyacentes son agregados en base a su ponderación, la cual refleja la estructura de correlación cruzada al variar el tiempo es decir el ciss pone más peso cuando hay estrés en varios mercados al mismo tiempo.

Se obtiene una matriz de ponderaciónes a partir de la función impulsorespuesta de los modelos var. Aquí se atribuye una ponderación a cada índice en función del impacto que ejerce en la producción industrial de la Zona Euro. El CIss aplica la teoría de porta- folios, dado que agrega diferentes indicadores de estrés individuales, cubre a los diferentes mercados financieros $y$ puede ser incorporado en los modelos macroeconómicos empíricos.

2. Indicador de integración financiera: SYNFINT. En abril de 2014 el BCE y la Comisión Europea publicaron cada uno un reporte donde se analiza el nivel global de la integración financiera en Europa gracias al nuevo indicador de integración financiera ${ }^{6}$. Este indicador refleja la evolución de cuatro de los principales mercados financieros: monetario, de bonos, bursátil y bancario. La metodología del sYNFIT es parecida a la del ciss, con excepción de que el indicador SYNFINT no mide la correlación ponderada cuando se agregan los índices de cada mercado financiero. El indicador SYNFINT es usado regularmente en los reportes del BCE sobre la integración financiera. El indicador SYNFINT da información tanto sobre el volumen de los intercambios financieros como sobre la homogeneidad de precios.

Este indicador sintético de integración financiera mostró que en Europa hay una fuerte fragmentación financiera tanto en la Unión Europea como en la Zona Euro. Gracias a este indicador se observó que en el año 2013 la estabilidad financiera mejoró como resultado de las medidas de política monetaria, principalmente de

${ }^{6}$ En inglés: Synthetic Indicator of Financial Integration (SYNFINT) 
las operaciones monetarias sobre títulos $\mathrm{OMT}^{7}$ y del avance en el proyecto de la Unión Bancaria Europea.

Este indicador mostró que en Europa se debe lograr una mayor integración en el mercado de obligaciones emitidas por el sector privado, el mercado bursátil y el mercado bancario. El nivel actual de fragmentación en los mercados financieros muestra un nivel similar a aquel que había antes de la introducción del euro. Se piensa que con las medidas de la Unión Bancaria Europea se va a poder alcanzar un nivel de integración financiera más elevado así como también con la introducción de reformas estructurales.

\section{Conclusiones}

Este trabajo de investigación constituye una contribución importante a la prevención de futuras crisis financieras. Sobre todo este trabajo otorgó un papel más importante a las interdependencias entre actores del mercado, al riesgo acumulado, al nivel de correlación del riesgo en diferentes mercados

\footnotetext{
${ }^{7}$ OMT: Outright Monetary Transactions. El BCE realizó compras masivas e ilimitada de títulos en los mercados secundarios de la deuda soberana sólo para los países que participaron en el Mecanismo Europeo de Estabilización (MEDE). Estas compras empezaron en el año 2012 y fueron abandonadas a principios del año 2015.

${ }^{8}$ Luna Flores, Valeria Ivett (2014).
}

financieros y a las discontinuidades de las variables económicas y financieras.

Disminuir e identificar con mayor precisión el riesgo sistémico agregado es uno de los principales desafíos de los bancos centrales y de la economía mundial. Como vimos el indicador CISs incluye al mercado cambiario mientras que el indicador SYNFIT no lo incluye. El indicador ciss es un indicador que mide el riesgo endógeno y el efecto contagio de 5 mercados financieros hacia la economía real. Se estima que el poder predictivo de ambos indicadores es muy bueno, ya que lograron reflejar la crisis financiera que comenzó en el año 2008.

Otro de los nuevos desafíos de esta nueva política macroprudencial es identificar los puntos fuertes y débiles de esta nueva serie de indicadores y modelos. Dentro de estas nuevas medidas macroprudenciales también se debe de considerar el papel que juegan las expectativas de los agentes económicos. El principal desafío es lograr un mayor grado de coordinación entre las políticas macroprudencial, monetaria y fiscal. 


\section{Referencias}

Banque de France (2014), “Amélioration de l'intégration des marchés financiers européens, qui demeure néanmoins inférieure au niveau d'avant la crise, selon deux rapports de la Commission Européenne et de la BCE”, 28 de abril.

BCE (2014), "La BCE publie les résultats définitifs des travaux du réseau de recherche dans le domaine macroprudentiel”, 23 de junio.

BCE (2014), Final Results of Macro-prudential Research Network, junio.

Berges, Angel (2013), "Riesgo sistémico", El País, 29 de octubre.

Guarda, Paolo, Abdelaziz Rouhabah y John Theal (2012), "An MvAr framework to capture extreme events in macro-prudential stress tests", working paper núm.1464, BCE, agosto.

Holló, Daniel, Manfred Kremer y Marco Lo Duca (2012), “CIss-a composite indicator of systemic stress in the financial system", working papers series núm.1426, ECB, marzo.

Luna Flores, Valeria Ivett (2014), "La actual restructuración del sistema de la Zona Euro", Economía Informa núm. 389, unam, noviembre-diciembre.

Trichet, Jean-Claude (2011), "Les défis intellectuels qui se posent à l'analyse de la stabilité financière à l'ère de la surveillance macroprudentielle", Revue de la Stabilité Financière núm. 15, Banque de France, febrero. 